\title{
Les coûts du mariage-les transactions matrimoniales dans le monde en développement
}

\author{
Sajeda Amin \\ Population Council \\ Ashish Bajracharya \\ Population Council
}

Follow this and additional works at: https://knowledgecommons.popcouncil.org/departments_sbsr-pgy

Part of the Demography, Population, and Ecology Commons, Family, Life Course, and Society Commons, Gender and Sexuality Commons, International Public Health Commons, and the Medicine and Health Commons How does access to this work benefit you? Let us know!

\section{Recommended Citation}

Amin, Sajeda and Ashish Bajracharya. 2011. "Les coûts du mariage-les transactions matrimoniales dans le monde en développement," Promouvoir la santé, la sécurité et la productivité transitions vers l'âge adulte Bulletin no. 35. New York: Population Council. 


\section{Les coûts du mariage - les transactions matrimoniales dans le monde en développement}

\section{Préparé par Sajeda Amin et Ashish Bajracharya}

A u cours des deux dernières décennies, le Population Council a recueilli une multitude d'informations sur les causes et les corrélats du mariage des enfants et documenté ses effets néfastes sur le bien-être des femmes et des filles. Dans de nombreuses régions du monde en développement, la compréhension du mariage des enfants est au cœur de la conception de programmes qui favorisent des transitions saines vers l'âge adulte. Les travaux de recherche du Population Council soulignent également l'importance des rituels et des pratiques d'échange matrimoniales dans le monde et éclairent les différences considérables entre ces pratiques. Ce bulletin fait la synthèse des principaux thèmes qui ont émergé des travaux de recherche sur les coûts du mariage. Alors même que les coutumes telles que la dot saisissent l'imagination populaire et l'attention des médias, il existe étonnamment peu de recherches universitaires ou des preuves empiriques pour jeter les bases de programmes et de politiques efficaces.

\section{Les transactions matrimoniales}

L'on entend par transactions matrimoniales les paiements ou les transferts de biens qui se produisent entre les familles de la mariée et du marié au moment d'un mariage. La forme, l'orientation et le montant des paiements varient largement. Dans certains contextes, comme dans les communautés hindoues ou sikhes du Népal ou en Inde du Nord, les négociations relatives à de tels paiements sont une pratique courante ; chez les musulmans d'Asie du Sud, en revanche, l'émergence des demandes de dot a soulevé colère et appréhension. La dot et le « prix de la fiancée » anthropologiques classiques (Goody et Tambiah 1973) apportent des définitions simples mais faisant autorité sur ces transactions comme indiqué ci-dessous :

Dot : Échange effectué par la famille de la mariée destiné à la famille du marié

Compensation matrimoniale / prix de la fiancée : Échange effectué par la famille du marié destiné à la famille de la mariée.

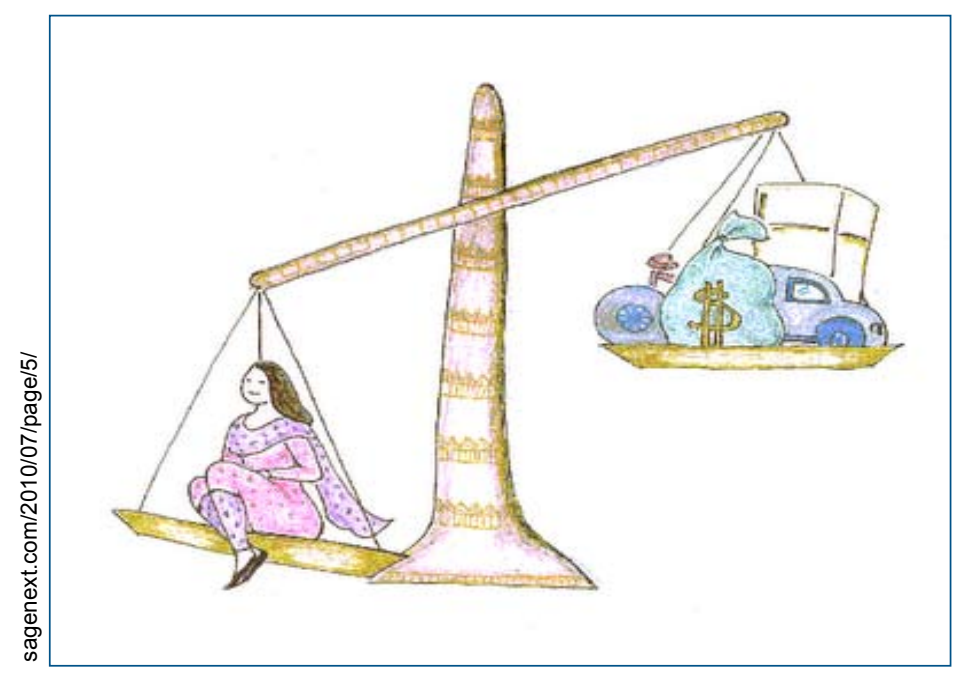

Entre ces deux types d'échange, il en existe une multitude d'autres qui se produisent pendant les mariages. Ceux-ci peuvent inclure la fourniture de services et l'échange de cadeaux ou de sœurs / filles entre les familles. Bien que n'étant pas ouvertement des transactions financières, ces échanges ont souvent une fonction d'échange économique sous-jacent. Les chercheurs en sciences sociales qui étudient les échanges matrimoniaux se penchent généralement sur le motif de ces transactions (par exemple, Caldwell, Reddy et Caldwell 1983 ; Becker 1991 ; Bhat et Halli 1999). Cependant, toutes les conséquences des transactions matrimoniales ne sont pas bien comprises. Les travaux menés par le Population Council avec des adolescentes suggèrent que des éléments tels que le choix du moment du mariage ou les pratiques comme le mariage des enfants et les frais occasionnés par un mariage peuvent avoir des implications importantes pour le bien-être post-matrimonial et la violence entre partenaires intimes. L"évaluation de la valeur d'une femme ou d'un homme sur le marché du mariage en termes de dot ou de « prix de la fiancée » peut avoir des conséquences particulièrement importantes pour l'estime de soi et le sentiment de confiance, avec des implications significatives pour le bien-être des adultes et 
l'égalité entre les sexes. Pourtant, les preuves recueillies sur les conséquences du coût financier du mariage restent extrêmement limitées.

\section{Traditions statiques et évolution des pratiques}

II y a tout lieu de penser que le problème repose sur la disparité des pratiques et l'évolution des attentes plutôt que sur des traditions établies de longue date. II est maintenant bien reconnu que les coûts du mariage se sont multipliés dans de nombreux environnements. Selon une série d'évaluations rigoureuses de données, l'avènement de la dot ou son augmentation au cours des dernières décennies est liée à la pression sur le mariage (un excès de filles à marier par rapport au nombre de garçons). Ce constat est particulièrement bien documenté en Asie du Sud. Au Bangladesh et en Inde, la période à laquelle les demandes de dots sont en hausse coïncide avec des taux élevés de croissance démographique, ce qui crée un déséquilibre dans le marché du mariage si l'on tient compte de la préférence culturelle pour une différence d'âge importante dans les mariages favorisant l'homme.

Dans de nombreux environnements, le mariage a désormais des coûts prohibitifs, sachant que les dépenses représentent plusieurs fois le revenu annuel total du ménage. Cette escalade financière peut être attribuée à des aspirations et à des niveaux de vie plus élevés. Lorsque ces attentes ne sont pas remplies, ces pratiques sont liées à différentes influences négatives sur le bien-être post-marital des femmes, qui sont victimes le plus souvent de formes graves de violence domestique.

Dans de nombreux cas, les transactions matrimoniales ne sont pas totalement rigides, mais relativement souples et liées à d'autres caractéristiques recherchées dans le marché du mariage. Lorsque le jeune âge d'une mariée est une condition recherchée, les familles pauvres peuvent décider de marier leurs filles à un âge précoce pour faire des économies sur le coût du mariage. À l'identique, des attentes inabordables en termes de « prix de la fiancée » peuvent encourager les jeunes à avoir des relations et des enfants en dehors du mariage. Les transactions fondées sur le mariage, que ce soit sous la forme de dot ou de "prix de la fiancée » exacerbent la marginalisation et l'exploitation des filles, sachant que ces deux pratiques banalisent et commercialisent la valeur de filles. Bien que le « prix de la fiancée » soit une pratique qui ressemble directement à une transaction servant à acheter les droits de reproduction et de production d'une fille, la dot perpétue également le cycle d'exploitation des filles.

\section{Les travaux du Population Council sur les transactions matrimoniales}

Les programmes du Population Council destinés à éviter le mariage des enfants (Amin, Chong et Haberland 2011) intègrent généralement des stratégies de développement des compétences des jeunes femmes axées sur des opportunités éducatives et l'apprentissage des modes de subsistance. Les travaux de recherche sur les dimensions économiques du mariage ont documenté ces approches programmatiques pour le bienêtre des filles. Les travaux du Population Council sur le mariage des enfants ont évolué pour intégrer des approches multisectorielles qui tiennent compte des considérations économiques du mariage. Étant donné que les traditions et les cultures matrimoniales, notamment les caractéristiques des transactions matrimoniales, varient même au sein de contextes nationaux, les approches programmatiques tentent de s'adapter à des contextes spécifiques. Quelques exemples sont présentés ci-dessous.

\section{La pauvreté et le choix du moment du mariage au Népal}

Selon une étude basée sur les données d'un panel national au Népal, un lien empirique clair a été établi entre le fait d'être pauvre pendant l'enfance et le fait de se marier au début de l'adolescence, plutôt que d'être scolarisé (Bajracharya et Amin 2010). Les demandes de dot ont augmenté ces dernières années. II est probable que ce lien empirique entre le moment du mariage et la pauvreté soit tempéré par les transactions matrimoniales. La presse populaire rapporte régulièrement des cas de demandes de dots élevées et les désaccords et violences qui en découlent. Pourtant, les pratiques de dot jouissent d'une certaine légitimité en tant que traditions séculaires et les critiques publiques ont parfois eu des conséquences désastreuses, telles que l'assassinat d'une journaliste de radio dans le sud du Terai, qui aurait été tuée à cause de ses déclarations publiques contre la dot. C'est dans les plaines du sud du Teraï au Népal que les demandes de dot sont les plus excessives.

\section{La grossesse pré-maritale, l'éducation et le « prix de la} fiancée » en Afrique du Sud

Le paiement du « prix de la fiancée » (lobola), fréquent dans les mariages en Afrique du Sud, symbolise le transfert du droit des filles à offrir des services sexuels et économiques à leur époux (Meekers 1992). Une étude menée en Afrique du Sud s'est penchée sur la grossesse pré-maritale et la parentalité à l'adolescence (Kaufman, de Wet et Stadler 2000). Elle présente la lobola comme un facteur important dans la détermination de l'éligibilité au mariage des filles qui ont eu des enfants. Sur la base de travaux de recherche qualitative, les chercheurs du Population Council ont conclu que l'attente du « prix de la fiancée » pousse les parents à investir dans l'éducation d'une fille et pourrait expliquer la tendance inhabituelle observée en Afrique du Sud, selon laquelle la plupart des filles retournent à l'école, même après l'accouchement. Les naissances hors mariage et les naissances pré-maritales pendant l'adolescence sont également liées à un « prix de la fiancée » plus faible en vue du futur mariage des filles, entraînant ainsi des circonstances où les naissances pré-maritales sont souvent gardées secrètes.

\section{La transformation du mariage et le comportement reproducteur au Ghana}

Une étude menée par des chercheurs du Population Council sur la nature changeante du comportement reproducteur au Ghana a examiné le rôle du « prix de la fiancée » pour expliquer la moindre importance du mariage et la hausse des relations sexuelles et de la maternité avant le mariage (Mensch et al. 1999). Les résultats qualitatifs suggèrent que les traditions nuptiales traditionnelles qui impliquaient un « prix de la fiancée » élevé étaient considérées comme un fardeau pour les jeunes hommes. Avec la baisse de la pratique de l'excision féminine, l'activité sexuelle avant le mariage est de plus en plus fréquente et la vie sexuelle 
des jeunes n'est plus confinée au sein du mariage traditionnel. Au fur et à mesure que les jeunes couples exercent un contrôle croissant sur les décisions de vie comme le mariage et la procréation, ils favorisent le contournement non seulement des coutumes du mariage traditionnel, mais également du poids financier au cours de ce processus. Les pratiques coutumières du « prix de la fiancée » élevé dissuadent les mariages officiels et contribuent à la disparition du mariage traditionnel et à la formation des familles dans cette région du Ghana.

Conséquences imprévues du retard de l'âge du mariage au Bangladesh Selon les données des Enquêtes démographiques et de santé (EDS), plus de 65 pour cent des femmes âgées entre 20 et 24 ans sont mariées avant 18 ans au Bangladesh. En 2001, le Population Council s'est associé avec plusieurs agences onusiennes et non gouvernementales au Bangladesh pour concevoir et évaluer un programme à large échelle consacré aux adolescentes, appelé Kishori Abhijan (Amin et Suran 2005). Kishori Abhijan est un programme axé sur la création d'espaces sûrs permettant aux filles d'avoir une vie sociale et de s'instruire, et offre une formation destinée à améliorer les compétences de subsistance. L'objectif était de contester les normes sociales rigides telles que le mariage précoce par l'autonomisation économique et sociale des filles. La surveillance des composantes du programme a montré que les volets du programmes concernant les espaces sûrs et le soutien social ont été appliqués de façon cohérente et qu'ils ont été bien acceptés. La formation relative aux moyens de subsistance, en revanche, s'est avérée coûteuse et difficile à mener à l'échelle. Par conséquent, seule une petite proportion de jeunes filles ont pu participer à cette formation. Une évaluation réalisée deux ans après le début du programme a révélé que même si le mariage avait été retardé pour les participantes au programme, le paiement de la dot a augmenté proportionnellement avec l'âge au mariage. Au Bangladesh, il existe une forte association entre l'âge au mariage et le montant de la dot payée (voir la Figure 1). Le programme a par la suite tenté de se pencher sur la question de la dot en travaillant avec les familles et a conclu que le problème était insoluble. Alors que les programmes offrant des espaces sûrs et un soutien social réussissent à re-

Figure 1 Montant moyen de la dot et âge moyen du mariage au Bangladesh

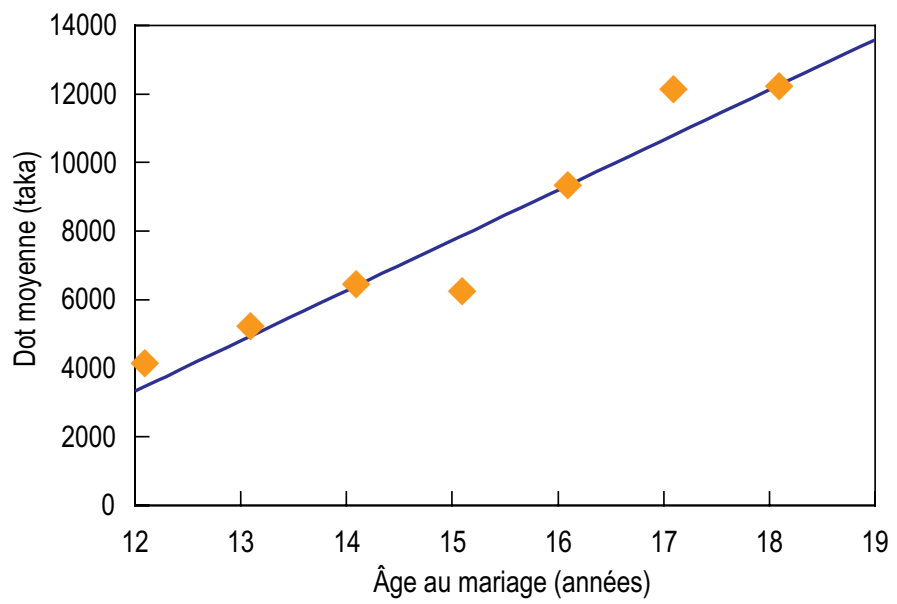

À partir des données du programme Kishori Abhijan sur les filles mariées entre 2001 et 2003. tarder l'âge du mariage, les demandes de dot proviennent des garçons et des hommes qui se marient. Dans une société où le mariage est très apprécié et détermine le statut social et économique des filles, le rejet des perspectives de mariage en raison des coûts de la dot n'est pas une option pour la plupart des familles.

Les données sur les transactions matrimoniales issues des travaux de recherche

En dépit de ces études, nos efforts pour comprendre les transactions matrimoniales et leur impact sur le bien-être des filles et des femmes ont été limités par un manque de données. Des enquêtes représentatives nationales menées auprès des ménages telles que les études sur la mesure des niveaux de vie (EMNV) de la Banque mondiale ou les Enquêtes démographiques et de santé (EDS) recueillent souvent des données uniquement sur l'âge au mariage et les caractéristiques du conjoint.

Parmi les enquêtes qui se penchent plus précisément sur le mariage et la formation de la famille, seules quelques-unes collectent des données sur une plus grande variété de variables matrimoniales. L'Enquête 2006 sur les normes de genre de la Banque mondiale menée au Bangladesh est exemplaire en termes de recueil de données détaillées sur les caractéristiques du mariage et les transactions matrimoniales (Banque mondiale 2008). Cet ensemble de données recueille des informations sur plusieurs thèmes liés au mariage que les chercheurs jugent essentiels pour mener des travaux de recherche empiriques rigoureux sur les transactions et la dynamique matrimoniales. Ces thèmes sont les suivants :

\section{Les conditions du mariage}

2. Le choix du conjoint

3. Les écarts de richesses et d'actifs entre les familles

\section{L'existence de l'endogamie villageoise}

\section{Les caractéristiques de la résidence post-maritale}

Les questions sur les transactions matrimoniales au Bangladesh de l'enquête de la Banque mondiale sont particulièrement bien détaillées. Les thèmes énumérés ci-dessous sur les transactions matrimoniales sont susceptibles de contribuer à mieux comprendre le rôle que les transactions matrimoniales jouent dans le choix du moment du mariage et la multitude de résultats et d'indicateurs relatifs au bien-être post-marital :

1. Y a-t-il eu un transfert d'argent ou de produits ?

2. Le montant de l'échange, monétaire ou autre

3. Le transfert est-il volontaire ou exigé par une famille ?

4. Comment les familles répondent aux demandes (y compris l'endettement) et les demandes dépassent-elles ce qu'une famille ordinaire peut payer?

5. L'existence de négociations sur ce qui doit être échangé et son montant et si un intermédiaire rémunéré a été impliqué

6. Comment les familles obtiennent les ressources financières pour payer une dot ou « le prix de la fiancée »? 
Les chercheurs du Population Council commencent à prendre conscience des différents types de données d'enquête quantitatives qui sont disponibles sur les transactions matrimoniales. Des études récentes suggèrent qu'il n'existe pas de données à grande échelle qui abordent ces thèmes et questions au niveau national. La plupart des travaux de recherche menés sur ce sujet proviennent invariablement de petits échantillons localisés et non représentatifs, réduisant considérablement la possibilité de généraliser les conclusions. Les initiatives qui encouragent la collecte de telles données auraient un impact positif sur les études relatives aux transactions matrimoniales au niveau national, avec un potentiel important pour assurer des recherches pertinentes dans le cadre de la prise de décision.

\section{Conclusions}

Bien que l'absence d'une base de données appropriée représente clairement un obstacle pour étudier les coûts du mariage de manière exhaustive, cette étude met en évidence plusieurs aspects importants sur les transactions matrimoniales. Nos travaux indiquent que dans nombreuses régions du monde le coût du mariage est le moteur du changement social. Même si les pratiques sont très variées, les conséquences pour les femmes sont majoritairement négatives. Alors que des programmes visant à promouvoir des transitions saines vers l'âge adulte émergent et se multiplient dans différents contextes, les manifestations spécifiques des coûts du mariage et de leurs corrélats doivent être prises en compte, tant en termes de compréhension du changement qui est déjà en cours que dans la conception de programmes qui apporteront un véritable changement.

Certains des exemples, tels que l'expérience au Bangladesh, montrent comment une compréhension nuancée peut éviter les conséquences imprévues de programmes qui ciblent une pratique spécifique telle que le mariage des enfants. Le cas du Népal souligne qu'il est important de cibler les pauvres, plus susceptibles d'être plus affectés par ces pratiques. D'autre part, les normes d'échange spécifiques en Égypte et au Pakistan pourraient avoir contribué à soutenir les traditions du mariage tardif pour les femmes. Les processus de développement et de modernisation, tels que l'urbanisation et la migration, pourraient bien saper la base de ces normes et abaisser davantage l'âge du mariage.

Les actions récentes, telles que l'approche utilisée dans les programmes de l'UNICEF qui tient compte des normes sociales, suggèrent qu'il s'agit d'un point de départ. En effet, il convient de trouver la dénomination de l'approche et d'insister sur le fait que des normes existent et doivent être abordées. En l'absence de preuves appropriées sur la définition, le fonctionnement et les conséquences des normes, il semble difficile de voir comment l'approche pourrait fonctionner.

\section{Bibliographie et publications connexes}

Amin, S., E. Chong et N. Haberland. 2011. "Programs to address child marriage: Framing the problem," Promoting Healthy, Safe, and Productive Transitions to Adulthood Brief No. 14. New York: Population Council.

Amin, S. et M. Das. 2009. "Marriage continuity and change in Bangladesh," document présenté au Séminaire sur le mariage, Indian Institute of Technology, New Delhi, 25-27 septembre (à paraître sous forme de chapitre dans un ouvrage).

Amin, S. et L. Suran. 2005. "Program efforts to delay marriage through improved opportunities: Some evidence from rural Bangladesh," Document présenté à l'Union internationale pour l'étude scientifique de la population (IUSSP). Tours, France, $18-23$ juillet.

Bajracharya, A. et Amin, S. 2010. "Poverty, marriage timing, and transitions to adulthood in Nepal: A longitudinal analysis using the Nepal Living Standards Survey," Document de travail n 19 du programme Pauvreté, Genre et Jeunesse. New York: Population Council.

Banque mondiale. 2008. "From whispers to voices: Gender and social transformation in Bangladesh," Bangladesh Development Series. Washington, DC: Banque mondiale.

Becker, G. 1991. A Treatise on the Family. Cambridge, MA: Harvard University Press.

Bhat, P.N.M. et S.S. Halli. 1999. "Demography of brideprice and dowry: Causes and consequences of the Indian marriage squeeze," Population Studies 53(2): $129-148$.

Caldwell, J., P.H. Reddy, et P. Caldwell. 1983. "The causes of marriage change in South India," Population Studies 37(3): 343-361.

Goody, J. et Tambiah, S.J. 1973. Bridewealth and Dowry. Cambridge University Press.

Kaufman, C.E., T. de Wet, et J. Stadler. 2000. "Adolescent pregnancy and parenthood in South Africa," Document de travail $n^{\circ} 136$ de la Division de la recherche en politiques. New York: Population Council.

Meekers, D. 1992. "The process of marriage in African societies: A multiple indicator approach," Population and Development Review 18(1): 61-78.

Mensch, B., D. Bagah, W. Clark, et F. Binka. 1999. "The changing nature of adolescence in the Kassena-Nankana district of northern Ghana," Studies in Family Planning 30(2): 95-111.

Suran, L., S. Amin, L. Huq, et K. Chowdury. 2004. "Does dowry improve life for brides? A test of the bequest theory of dowry in rural Bangladesh," Document de travail no 195 de la Division de la recherche en politiques. New York: Population Council.

12 Population Council

Le Population Council fait évoluer les perspectives sur les questions essentielles de santé et de développement. Nous cherchons à comprendre les causes et les conséquences de l'inégalité entre les sexes et des disparités sociales qui s'installent au cours de l'adolescence. Nous fournissons des données en vue d'améliorer les programmes et les politiques mis en place sur le terrain qui permettent d'assurer des transitions efficaces et productives vers la vie adulte dans les pays en développement. www.popcouncil.org

(C) 2011 The Population Council, Inc

4 - Rendez-vous sur www.popcouncil.org/publications/serialsbriefs/TABriefs.asp pour consulter tous les bulletins d'information de la série Promoting healthy safe, and productive transitions to adulthood [Promouvoir des transitions saines, sécurisées et productives vers l'âge adulte]. 